Group-Based Trajectory Modeling of Fear of Disease Recurrence Among Women

$$
\text { Recently Diagnosed with Gynecological Cancers }
$$

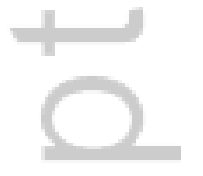

a

3

C

\section{$4 \cdot 9$}

$\longrightarrow$

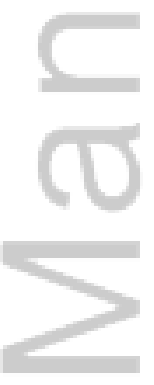

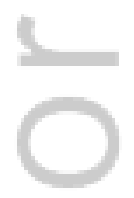

\author{
Sharon L Manne, Ph.D. \\ Rutgers Cancer Institute of New Jersey \\ 195 Little Albany Street, New Brunswick, NJ 08903
}

$\begin{array}{ll}\text { P. 732-235-6759 } & \text { F. 732-235-6225 }\end{array}$

mannes1@ cinj.rutgers.edu

Shannon Myers-Virtue, Psy.D.

Temple University School of Dentistry

David Kissane, M.D.

Memorial Sloan Kettering Cancer Center and Monash University

Melissa Ozga, D.O.

Memorial Sloan Kettering Cancer Center

Deborah A. Kashy, Ph.D.

Michigan State University

Stephen Rubin, M.D.

Fox Chase Cancer Center

Carolyn J. Heckman, Ph.D.

Fox Chase Cancer Center

Norman Rosenblum, M.D.

Thomas Jefferson University

We would like to acknowledge project managers Tina Gajda, Sara Worhach, Shira Hichenberg, and Kristen Sorice, assistant project manager Jaime Betancourt, and research study assistants Joanna Crincoli, Katie Darabos, Sloan Harrison, Travis Logan, Glynnis McDonnell, Arielle Schwerd, Marie Plaisime, and Caitlin Scally. We would also like to thank the study participants, their oncologists, and the clinical teams at Rutgers Cancer Institute of New Jersey, Memorial Sloan Kettering Cancer Center, Fox Chase Cancer Center,

This is the author manuscript accepted for publication and has undergone full peer review but has not been through the copyediting, typesetting, pagination and proofreading process, which may lead to differences between this version and the Version of Record. Please cite this article as doi: $10.1002 /$ pon.4223

This article is protected by copyright. All rights reserved. 
Trajectories of Cancer Fears

the Hospital of the University of Pennsylvania, Thomas Jefferson University, Morristown Medical Center, and Cooper University Hospital.

Source Funding: This work was funded by NIH grant R01 CA085566 to Sharon Manne with the National Cancer Institute.

Disclosure information: Nothing to disclose
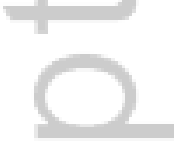

\section{ABSTRACT}

Objective: Fear of cancer recurrence is an important clinical phenomenon and is associated with decrements in life domains. The study goals were to characterize patterns of global fear of recurrence (FOR) and four domains of fear (health, role, womanhood, and death worry) over time in women who were diagnosed with gynecological cancer and to identify demographic, medical, and psychological predictors of FOR.

Method: One hundred eighteen women participating in the Usual Care arm of a randomized trial completed the Concerns about Recurrence scale as well as measures of depressive symptoms, cancer-specific distress, coping, coping efficacy, and social network responses at four time points. The majority of the sample was diagnosed with stage 3 ovarian cancer.

Results: Group-based trajectory modeling identified subgroups of women with high-stable (49.1\%), high-decreasing (25.3\%), and low-stable (25.5\%) trajectories for global FOR. For role worries, three similar group trajectories were identified. For health worries, modeling identified subgroups with high-decreasing (19.1\%) and low-increasing (80.9\%) trajectories. For womanhood worries, modeling identified subgroups with high-increasing (15.7\%) and low-decreasing (84.2\%) trajectories. Young age, metastatic cancer, depression, cancer 


\section{Trajectories of Cancer Fears}

distress, holding back, and lower coping efficacy were associated with the high-stable global FOR and at least one domain of FOR.

Conclusion: Almost half of the women recently diagnosed with gynecological cancer

evidence persistently elevated FOR over the six month period post-diagnosis. Psychological interventions to reduce FOR may be more effective if they focus on teaching patients coping skills, as well as greater comfort expressing cancer-specific concerns to others.

Keywords: Recurrence fear, gynecological cancer, emotional expressivity, psychological adaptation, coping with cancer

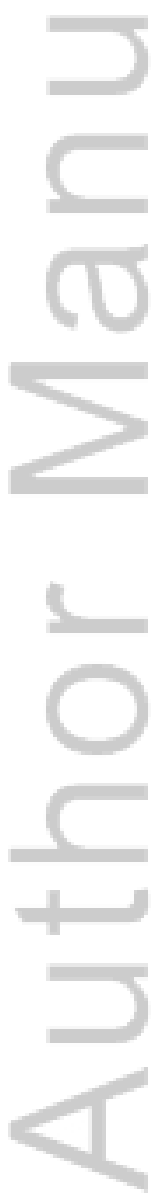

This article is protected by copyright. All rights reserved. 
Trajectories of Cancer Fears

Fear of cancer recurrence (FOR) is a pervasive concern among cancer patients $[1,2]$. FOR encompasses a variety of illness-related fears and is defined as the fear that cancer will recur, progress, or metastasize in the same or another part of the body $[3,4]$. There is a large quantity of literature suggesting that between $17 \%$ and $72 \%$ of cancer patients report elevated levels of FOR [5-7].ad persists in a subgroup of survivors [8]. Evidence suggests that elevated levels of FOR persist among a subgroup of cancer survivors many years after treatment has been completed [8]. Because of the large percentage of patients who experience it, FOR may be considered a normal response to the experience of a lifethreatening illness such as cancer.

\section{The Course of FOR over the Cancer Trajectory}

The majority of longitudinal studies suggest that FOR declines among the majority of cancer patients in the first year after diagnosis [9-11]. However, its course is highly variable, with individual differences over time $[12,13]$. Some patients' FOR remains stable, with either consistently elevated, consistently moderate, or consistently low levels of fear $[2,10,14]$.

FOR as a Multi-dimensional Construct

Although FOR has consistently been associated with distress and lower quality of life $[15,16]$, there has been less attention given to its complexity. Two conceptualizations of FOR $[15,16]$ propose that it is a multidimensional construct. These formulations illuminate the nature of fears and illuminate what aspects about possibility of recurrence or progression of 


\section{Trajectories of Cancer Fears}

cancer cause fear. Thus, global fears are "unpacked" or separated into the domains of life and future events (e.g., death) that contribute to the fear experience. These domains may include fear about the impact of recurrence on sexuality and romantic relationships, concerns about the possibility of future medical treatments, concerns about the impact of future emotional upset, fear about being able to fulfill work and home responsibilities, and worry about the possibility of death [16]. By considering FOR as a multidimensional construct, the complexity of this construct can be more fully understood.

\section{Correlates and Predictors of FOR}

Cross-sectional studies have indicated that FOR is associated with lower mental, physical, and social aspects of quality of life [7,17 ],greater health care utilization, [18] anxiety [5], depressive symptoms [19], and intrusive thoughts [12,20]. Longitudinal studies have suggested that demographic, medical, and psychological factors contribute to increases or decreases in FOR over time. Younger age, [21] lower socioeconomic status[10], advanced disease [2], elevated initial depression[10], and elevated anxiety [12] are associated with elevated FOR [10]. In addition, less social support and more negative social interactions have been associated with higher FOR over time [10]. Holding back sharing concerns is associated with elevated FOR in cross-sectional research [22]. [13]. While coping strategies have been emphasized to a lesser degree in the literature, one study does suggest that lower coping self-efficacy is associated with elevated FOR among breast cancer survivors [13]. The Present Study 
Trajectories of Cancer Fears

Most longitudinal studies describe average changes in FOR over time. These analyses are useful, but more can be learned by identifying subgroups of women who exhibit common patterns of change over time. For example, women who begin with high FOR that decreases may differ with regard to their characteristics as compared with women who begin with low FOR and remain stable. Group-based trajectory analyses can be used to identify subsets of persons who exhibit similar longitudinal patterns in FOR, providing a more detailed understanding than what is possible with typical longitudinal analyses, particularly when four or more time points are used [23]. Understanding changes in FOR and its domains over time and the predictors of these changes can help clinicians identify who is in need of psychological intervention and how to effectively intervene. Towards this end, our primary goal was to determine whether there are distinct patterns of FOR with stable, increasing, and decreasing trajectories. We examined trajectories of FOR in a patient population that has not been well-studied in longitudinal research, women who are newly diagnosed with gynecological cancers. The diagnosis and treatment of gynecologic cancer has considerable physical and psychosocial impact $[24,25]$. There is relatively little known about the prevalence and course of FOR among women diagnosed with this cancer, despite the fact that many types of gynecologic cancers have high recurrence rate [26]. Between one-fifth and one-half of women with these cancers report elevated FOR [27, 28]. We proposed that there would be varying patterns of FOR with stable, increasing, and decreasing patterns. We proposed two possible trajectories: the majority of participants' FOR would decline over the 
six month period after diagnosis [10], but there would be a group who was characterized by persistently elevated FOR.

The second goal was to evaluate the demographic, medical, and psychological factors associated with FOR trajectory subgroups. We predicted that being younger, being diagnosed with more advanced disease, and reporting greater baseline physical impairment would characterize the group of patients reporting persistently elevated FOR. We evaluated three categories of factors: distress, cancer-related coping strategies and cancer-related coping efficacy, and difficulties with one's social network. We proposed that women with persistently elevated FOR would have higher baseline depression and cancer-specific distress. Based on theories regarding coping with traumatic events [29], we proposed that approach-oriented coping strategies (positive reappraisal, problem-solving, expressing emotions) and greater coping self-efficacy would predict declines in FOR over time. We proposed that women who reported less holding back sharing concerns and fewer unsupportive responses would evidence declines in FOR over time. There has been little work that has evaluated variables associated with group-based trajectories of FOR, making it difficult to specify further the expected relationships of variables with global FOR and its domains over time.

\section{METHOD}

\section{Participants and Procedures}


The sample consisted of women who were newly diagnosed with primary gynecological cancer (ovarian, endometrial, cervical, fallopian tube, or uterine cancer) and undergoing active medical treatment (defined as having surgery, chemotherapy, and/ or radiation) at seven hospitals in the northeastern US. They were enrolled in a randomized clinical trial evaluating the efficacy of a Coping and Communication Intervention versus a Supportive Counseling Intervention and Usual Care [30]. Study inclusion criteria were: age 18 years or older; diagnosed with the cancer within the past six months at the time of recruitment, a Karnofsky Performance Status of 80 or above or an Eastern Cooperative Oncology Group score of 0 or 1 ; lived within a two-hour commuting distance from the recruitment center; English speaking; and no hearing impairment. Eligible women were identified and contacted. Participants signed an informed consent document approved by an Institutional Review Board.

Of the 1247 eligible patients approached, 351 consented to the larger randomized clinical trial $(28.1 \%)$. Of these, 118 patients were assigned to the Usual Care arm and included in this study. Because subjects in the present study are a subset of those who participated in the larger trial, we will present refuser information from the larger trial. Thus, the percentages presented are from all women approached for the larger study who refused the larger trial. . Among the 350 refusers of the larger trial, 27.1\% were passive refusers and could not be reached to refuse or accept participation. Among those patients providing a 


\section{Trajectories of Cancer Fears}

reason, most common reasons provided were: did not feel they would benefit (17.3\%), too overwhelmed (12.2\%), too much time to participate $(9.8 \%)$, and too ill to participate $(4.7 \%)$.

Comparisons between study participants and refusers of the parent study indicated that participants were younger $\left[\mathrm{t}(1028)=3.3, \mathrm{p}<.01, \mathrm{M}_{\text {participants }}=56.4, \mathrm{M}_{\text {refusers }}=60.3\right.$ years] and diagnosed more recently $\left[\mathrm{t}(1023)=2.9, \mathrm{p}<.01, \mathrm{M}_{\text {participants }}=3.9, \mathrm{M}_{\text {refusers }}=4.5\right.$ months]. The acceptance rate was significantly higher at two sites than the other four sites (Chi-square $(6)=55.6, \mathrm{p}<.001)$, due to the fact that the nurse practitioner approached patients about the study first at two of the sites. There were no differences with regard to type of cancer (ovarian vs. other), stage of disease, and race/ethnicity.

Surveys were administered at baseline (Time 1), five weeks (Time 2), nine weeks (Time 3), and six months (Time 4) after the baseline survey which corresponded to key time points in the larger intervention trial. Of the 118 participants completing a Time 1 survey, $107(90.6 \%)$ completed the Time 2 survey, 110 (93.2\%) completed the Time 3 survey, and $100(84.7 \%)$ completed the Time 4 survey. Participants were allowed to choose to skip an assessment and complete a later assessment. Because the analytic approach used all available data, all participants were included in the analysis.

\section{Outcome Measure: Fear of Recurrence/Progression}

The Concerns about Recurrence Scale [16] is a 29-item measure of the extent and nature of fear of breast cancer recurrence. We adapted the scale for the current study by asking about a recurrence of gynecological cancer. The scale contains four items assessing 
Trajectories of Cancer Fears

global fears (e.g., "How much time do you spend thinking about the possibility that your gynecologic cancer could recur?'). Ratings for the global fears items ranged from 1 (I don't think about it at all) to 6 (I think about it all the time). Four subscales assessed worry about health issues (10 items, "Interfere with my ability to plan the future"), womanhood issues (7 items, "Make me feel badly about how my body looks or feels"), role issue (6 items, "Keep me from fulfilling important roles in my job or at home"), and death worries (2 items, "Threaten my life")(scores range from 0-8). The stem for each domain item was, "I worry that a recurrence of gynecological cancer would..." Ratings for the domains ranged from 0 (not at all) to 4 (extremely). Internal consistency for all five scales has been demonstrated in two studies focusing on breast cancer patients $[7,16]$, but there is no prior work with gynecological cancer patients. In the current study, internal consistency for the scales ranged from .85 to .94 across time points.

\section{Baseline Predictors}

Depressive symptoms. The Beck Depression Inventory [31] is a 21-item scale used to assess depressive symptomatology. Internal consistency was .81 .

Cancer-Specific Distress. The Impact of Events Scale [32] is a 15-item measure of intrusive thoughts, avoidance, and numbing. Internal consistency was .89.

Positive reappraisal and planful problem solving. Four items assessed positive reappraisal coping and 4 items assessed problem solving [33]. Participants were asked to 
rate the ways they were coping with the cancer experience. Ratings ranged from $1=$ Did not do this, to $4=$ Did this a lot. Internal consistency was .88 .

Emotional expressivity. Emotional expressivity is a measure of a person's general tendency to express emotions. This construct was assessed using the Emotional Expressivity Questionnaire which is a 16-item scale measuring the level of comfort with expressing emotions [34]). Sample items are "When I really like someone they know it" and "I often tell people that I love them." Items are responded to on a 5-point Likert scale, with $0=$ never, and $4=$ always. Scale scores can range from 0-64. Internal consistency was .77. Cancer-specific coping self-efficacy. A 17-item scale was adapted from the Stanford Inventory of Cancer Patient Adjustment [35] for this population to assess self-efficacy beliefs for challenges for gynecological cancer patients. Items assessed the ability to communicate openly, manage anxiety, and manage affective responses. Participants were asked to rate confidence in managing each situation $(0=I$ cannot do this at all to $5=\mathrm{I}$ can do this extremely well). Internal consistency was .94 .

Holding back sharing concerns with family and friends. Holding back sharing concerns was assessed utilizing a previously-developed measure [36], which is 12 cancer concerns rated on a 6 -point scale $(0=$ not at all to $5=a$ lot $)$. Higher scores indicate more holding back sharing. Because participants did not rate holding back sharing concerns if they did not report having a concern, an average was used in the analysis. 
Perceived unsupportive responses from family and friends. A 13-item scale adapted from the Cancer Support Inventory [37] assessed unsupportive responses from family and friends rated on a four point scale $(1=$ never responds this way to $5=$ often responds this way). Internal consistency was .89.

Physical impairment. The 26-item physical limitations subscale of the Cancer Rehabilitation Evaluation System [38] assesses difficulty completing daily tasks and activities. Participants rated impairment during the past month from 0 (not at all) to 4 (very much). Internal consistency was .92.

Medical status. A medical chart review was used to capture primary cancer diagnosis, disease stage, metastatic status, treatments received (surgery, chemotherapy, and/or radiation), and time since diagnosis. For the analyses, the number of treatments received was entered into the equation rather than the treatments received. As can be seen in Table 1, the majority received surgery and chemotherapy. Thus, categorizing treatments received would not yield meaningful results.

\section{Analytic approach}

The PROC TRAJ procedure in SAS (version 9.4) was used to conduct a group-based trajectory modeling approach, which is a specialized application of finite mixture modeling [39]. In a non-technical sense, this approach is a combination of multilevel growth modeling and cluster analysis in that it is designed to identify subgroups of individuals who show similar trajectories on an outcome over time. Time was coded such that a 1 unit change 
corresponded to a 1 month increase, and because each woman completed and returned the questionnaires at somewhat different times in spite of the fact that questionnaires were sent at 5 and 9 weeks and again at 6 months, we used the woman's date of questionnaire return as our measure of time. On average we received the questionnaires at $1.73,2.86$, and 6.35 months after baseline, which was coded as 0 months. Thus, the intercept refers to the initial assessment. Models were developed by sequentially testing 1, 2, 3, and 4 subgroup models, initially allowing for linear and quadratic fixed effects for time. However, because there were no significant quadratic effects, the final models only included intercepts and the linear effect of time. A common random intercept variance was specified across the subgroups, and maximum likelihood was used as the estimation method.

The PROC TRAJ procedure generates group membership classification for each participant in the study, and we used that information to examine the extent to which there were differences between the subgroups on demographic, medical, and psychological variables. With regard to group membership criteria, trajectories were labeled "stable" when the linear effect was not significantly different from zero. Trajectories were labeled "increasing" when the beta was significantly different from zero and the sign for the coefficient was positive. Trajectories were labeled "decreasing" when the beta was significantly different from zero and the sign for the coefficient was negative.

We used SPSS Version 22 to conduct ANOVAs on quantitative variables to assess mean differences across subgroups, and significant ANOVAs were followed-up using post- 
Trajectories of Cancer Fears

hoc Tukey tests to correct for family-wise error. We used chi-square tests to evaluate subgroup differences on categorical variables.

\section{RESULTS}

\section{Descriptive Information on the Sample}

Table 1 contains characteristics of the sample. The majority was Caucasian $(79 \%)$ and married (69\%). The average age was 56.2 years (range $24-80$ years), and the majority of the sample was diagnosed with ovarian cancer (53\%). Slightly less than half (41\%) of the sample had metastatic cancer and were diagnosed with stage III disease (48.3\%). The average time since diagnosis was 3.9 months. The majority had undergone surgery and were receiving chemotherapy at the time of the baseline survey. Table 2 contains information about global FOR and the four domains and the baseline predictors.

\section{Trajectory Analysis Results}

Table 2 presents the trajectory results for global FOR, role worries, health worries, and worries about womanhood over time. Worries about death are not included in the table or in subsequent analyses because the trajectory analyses for that variable did not converge regardless of the number of subgroups specified in the model. The BIC values, along with statistical significance tests of the model parameters indicated that the best models for global fear and role worries consisted of three subgroups. The best models for health and womanhood worries included two subgroups. Graphs of the subgroup trajectories for the four fear variables are presented in Figure 1. 
Trajectories of Cancer Fears

For global FOR the first subgroup, comprising $25.5 \%$ of the sample, was low in FOR at the first assessment and stayed low over time (Low-stable). The second subgroup, comprising $25.3 \%$ of the sample began the study with relatively high global fear, but that fear decreased over time (High-decreasing). The final subgroup was the largest, with $49.1 \%$ of the sample. Women in this subgroup began the study with high global fear that remained high over time (High-stable).

Role worry subgroups were similar to those for global FOR. The first subgroup, $33.4 \%$ of the sample, started and stayed low in role worries (Low-stable). The second subgroup started at moderate role worries and then decreased over time (40.2\%) (Moderatedecreasing), and the final group started high in role worries and stayed high over time (26.4\%)(High-stable). For health worry, the first subgroup started low but increased somewhat over time (19.1\%)(Low-increasing). The second group started high but decreased somewhat over time (80.9\%) (High-decreasing). Finally, for womanhood worries the first subgroup started low and decreased over time (84.3\%)(Low-decreasing). The second subgroup started high and increased over time (15.7\%)(High-increasing).

\section{Characteristics Related to Trajectory Subgroup Membership}

We examined whether the subgroup classifications differed as a function of demographic variables including patient ethnicity (white/non-white), marital status (married/not married), income, and age. For the categorical variables, we used chi-square tests. For the quantitative variables, we used ANOVA to test for subgroup differences. 
Although there were no significant associations between trajectory subgroups and income or marital status, age and ethnicity were related to the subgroups. Specifically, there were significant age differences for role worries and womanhood worries. Post-hoc Tukey tests indicated that for role worries, women who were in the High-stable subgroup were significantly younger than women in the Low-stable subgroup. Women in the middle group (Moderate-decreasing) were not significantly different from the other subgroups. Likewise, women in the High-increasing subgroup for womanhood worries were younger than women in the Low-decreasing subgroup. There were also associations between ethnicity (white/nonwhite) and both global FOR subgroups and health worries subgroups. For global FOR, nonwhite women were most likely to be in subgroup 1 (Low-stable), but white women were most likely to be in subgroup 3 (High-stable). For health worries, both white and non-white women were more likely to be in the High-decreasing subgroup, but this tendency was especially strong for white women.

We also tested for subgroup differences on medical variables. Only functional impairment and metastatic status differed as a function of the subgroups. Differences in functional impairment were found for the role worry subgroups and the womanhood worry subgroups. Women in the High-stable (subgroup 3) for role worry reported significantly higher functional impairment than women in the Low-stable subgroup. Similarly, women in the High-increasing womanhood worry subgroup (subgroup 2) reported significantly higher functional impairment than women in the Low-decreasing womanhood worry subgroup. For 
Trajectories of Cancer Fears

metastatic status, there were significant subgroup differences for both global FOR and womanhood worries. The frequencies indicate that women without metastatic cancer were more likely to be in the Low-stable global FOR subgroup and women with metastasized disease were more likely to be in the High-stable global FOR subgroup. The pattern for womanhood worries was somewhat different in that women with metastasized cancer were especially likely to be in the Low-decreasing womanhood worry subgroup.

Table 3 presents the means, standard deviations, and F-tests assessing the extent to which women in the different subgroups for global FOR and the three worry variables differed in their initial psychological functioning and coping strategies. No significant differences between the subgroups were found for initial emotional expressiveness, problem solving coping, or unsupportive responses from friends and family. These variables are not included in Table 3 and are available from the authors. There were significant differences for initial cancer-specific distress, depression, holding back sharing concerns, coping selfefficacy, and positive reappraisal coping.

As an overview of the pattern of results, in every case in which there were statistically significant mean differences across the subgroups, regardless of the outcome variable, the differences between the lowest fear subgroup (i.e., subgroup 1 which was Low-stable) and the highest fear subgroup (i.e., generally High-stable or subgroup 3 for global FOR and role worries and subgroup 2 for health and womanhood worries) were significant. The middle subgroup for global FOR and role worries (i.e., starts High-decreasing) generally did not 
Trajectories of Cancer Fears

differ from the High-stable subgroup. More specifically, women in the High-stable subgroup for global FOR (subgroup 3) were significantly higher in initial cancer-related distress and initial depression, and they reported holding back sharing concerns to a greater degree than women in the Low-stable subgroup (subgroup 1). They also reported significantly lower initial coping efficacy. Similarly, women in the High-stable subgroup (subgroup 3) for role worry reported higher initial cancer-related distress, depression, and lower coping efficacy than women in the Low-stable subgroup (1). Women in the High-decreasing subgroup for health worries were significantly higher than the Low-increasing subgroup in initial cancerrelated distress, depression, and holding back sharing concerns, and they were significantly lower in initial coping efficacy and positive reappraisal coping. Finally, women in the Highincreasing womanhood worry subgroup were significantly higher in initial distress, depression, and holding back, and they were significantly lower in coping efficacy.

\section{Discussion}

To our knowledge, this study was first to examine group trajectories of FOR and its domains across a six month time period following initial diagnosis of gynecological cancer and to examine the demographic, medical, and psychological factors associated with these group trajectories. Three major findings warrant discussion. First, there were subsets of patients with different trajectories of global FOR and three of the four domains of fear examined, and the FOR distribution within each group trajectory differed across global FOR and the domains. Group trajectories were similar for global FOR and role worries and consisted of three groups, but trajectories for the health and womanhood worries were less 
Trajectories of Cancer Fears

complicated. The percentage of the sample in each group trajectory differed when global FOR and health domain worries were compared with role and womanhood worries. Almost half of the sample reported High-stable global FOR over time, and more than $80 \%$ reported High-decreasing health worries. In the role and womanhood worry domains, a lower proportion reported High-stable or High-increasing worries. For these outcomes, most women experienced either a decline in worry or Low-stable worry. Overall, across global FOR and its domains, a significant proportion continued to experience high FOR, with higher elevations shown in the global FOR and health domains than the role and womanhood domains. These results suggest that adopting a multidimensional perspective on FOR may elucidate a more nuanced understanding of patterns of change in FOR and specific domains of worry which remain elevated over time.

A second notable finding was that a number of psychological, coping, and support network variables differentiated the subgroup trajectories, but many did not. The strongest differences between FOR trajectories were noted for initial depression and cancer-related distress, such that the High-stable trajectory group differed from the lowest trajectory groups. Notably, women in the High-stable group evidenced lower coping efficacy than women in the other two groups. For three of the four FOR outcomes, women in the High-stable or increasing FOR group trajectories reported more holding back sharing concerns than women in the low FOR trajectories. There were no significant differences between the subgroups for general emotional expressiveness, problem solving, or perceived unsupportive responses. It 


\section{Trajectories of Cancer Fears}

is possible that our measure of emotional expressiveness captured the general tendency to share of non-cancer related feelings (e.g., "I am so glad you are in my life"), but the measure did not capture the degree to which women express their fears about recurrence with others. Problem-solving coping is likely not beneficial for managing fears about cancer recurrence, which cannot be controlled or solved. Unsupportive responses may not be associated with FOR because our measure assessed critical or avoidant reactions surrounding the patient's cancer, but we did not specifically assess unsupportive reactions related to the disclosure of fears about cancer recurrence (e.g., "Seemed uncomfortable talking to you about your illness" was assessed rather than "Seemed uncomfortable talking to you about your fears about cancer coming back").

Overall, these findings are consistent with cross-sectional and longitudinal research suggesting that depression is associated with global FOR [10,12] and extends this work by suggesting that depression as well as cancer-specific distress are associated with fears about the impact of cancer progression on specific domains of life. Holding back sharing concerns has been associated with global FOR in our prior cross-sectional work [22], and has been consistently associated with psychological distress in a number of prior studies with patients dealing with other kinds of cancer [36]. As noted by Lepore [29], sharing concerns may facilitate adaptation by providing the patient an opportunity to process her fears with others and allow others to help her challenge her negative beliefs about her future. Little attention has been paid to coping self-efficacy among women with gynecological cancer, but coping 
Trajectories of Cancer Fears

self-efficacy has been associated with lower global FOR among survivors of other types of cancer $[13,40]$. This pattern suggests that improving patients' confidence that they can manage cancer stressors and assisting patients with sharing concerns may assist patients who endorse high levels of global FOR and role and womanhood worries. Facilitating positive reframing of the cancer experience using traditional cognitive-behavioral therapy or third wave cognitive-behavioral techniques such as Mindfulness- Based Stress Reduction and Acceptance and Commitment Therapy [41] may be helpful for women with elevated health domain worries. Teaching problem solving skills, focusing on dealing with unsupportive family and friends, and increasing general levels of emotional expression (e.g., not necessarily emotions associated with the fear of cancer recurrence) may not be as effective for women reporting high FOR.

The third notable finding was the inconsistent role of demographic and medical factors. Women in the High-stable global FOR trajectory group were more likely to be White. This is the first time that race has been reported as a correlate of global FOR. Given that the majority of the sample was Caucasian, the finding cannot be generalized, and further research is needed. Women in the High-increasing womanhood worry and High-stable role worry groups were more likely to be relatively young. Due to normative life expectations, younger women may place more emphasis on body image, sexuality, childrearing, and employment roles, and younger women may be more distressed by the threat of recurrence on those areas. Among the medical predictors, physical impairment and metastatic disease 
Trajectories of Cancer Fears

were associated with group trajectories with different patterns for global FOR and its domains. Women who had High-stable global FOR were more likely to be diagnosed with metastatic cancer. Prior work has suggested that advanced stage cancer is associated with elevated FOR [2,22]. The pattern for womanhood worries differed: the Low-declining womanhood worry group was more likely to have metastatic cancer than the High-increasing group. One possible explanation for this finding is that women with advanced disease may worry less about the impact of the disease on their body image and sexuality because the impact on other areas of their lives is more significant. When functional impairment was examined, there were differences with regard to role and womanhood worries. Women in the High-stable role worry group reported higher initial functional impairment than women in the Low-stable role worries group, and women in the High-increasing womanhood worry group reported higher functional impairment than women in the Low-decreasing womanhood worry group. Thus, women experiencing higher levels of initial physical impairment are more likely to report consistently elevated concerns about their abilities to fulfill their work and family responsibilities and possibly trigger worries about sexual functioning and body image. There are several limitations to note. The first limitation is that the sample consisted of Usual Care participants from a randomized clinical trial. Thus, we did not conduct a population-based study assessing the natural course of FOR. Women participating in a psychotherapy trial may be more distressed and experience fewer fears about recurrence than women who decline participation. Therefore, the design used in this study may introduce 


\section{Trajectories of Cancer Fears}

important biases in our results, which may include regression to the mean and the effect of being in a clinical study. A second and related limitation is the participation rate $(28.1 \%)$. Although this participation rate is common for a psychotherapy trial, this rate is relatively low for an observational study. As noted above, women who decline an intervention study may be less distressed, endorse fewer fears about cancer recurrence, and possess more coping and support resources than women who accept participation. A study that was designed as an observational study may have yielded a lower proportion of participants in the High-stable global FOR trajectory and a greater proportion of participants in the Low-stable global FOR trajectory, and similar patterns in the role, health, and womanhood worry subgroup trajectories.

Third, the sample size of trajectory subgroups that were less than $25 \%$ of the sample were quite small ( $n=18-25$ each). Fourth, the small number of measurement time points may have affected the trajectory shape and group membership. In future research, a longerterm assessment should be conducted to assess whether the patterns observed within six months persist over longer periods of time, when disease progression is likely to occur, particularly among women diagnosed with ovarian cancer. A fifth and related limitation is that the time points were chosen to correspond to the intervention timeline for the larger intervention trial, and may not be ideal for the time course of FOR. Future studies may consider assessing FOR at time points that correspond to meaningful disease transitions, such as the end of definitive treatment and/or one year being cancer-free. Sixth, the majority of 
Trajectories of Cancer Fears

sample was diagnosed with ovarian cancer. Samples that include a larger proportion of women with other types of gynecologic cancers would clarify whether there are differences between different cancers. Additionally, the cohort was a relatively small sample of mostly Caucasian, well-educated, and middle-class patients. Future research might focus on minority and less socio-economically advantaged patients. Seventh, women who chose to participate were significantly younger and diagnosed more recently, and thus it is possible that the age differences that emerged in regard to FOR reflected the fact that the sample was younger than the general population of women with gynecological cancers. Finally, there were limitations of our measures. Our measure of cancer-specific distress, the IES, is a measure of subjective distress caused by a traumatic event (in this case, cancer). Items assess intrusive thoughts and avoidance of distressing cancer-related thoughts and feelings. However, this measure may not capture all aspects of cancer-related distress. In addition, the measure of depressive symptoms contains several items which assess somatic symptoms that may be associated with cancer treatment (e.g., reduced appetite, fatigue) and thus may have some overlap with recurrence fears, particularly fears about health. General emotional expressivity was assessed in this study, rather than expression of feelings and fears about the cancer experience. Although we did assess holding back sharing cancer-related concerns, future studies may benefit from including the degree to which the patient expressed cancer-related fears to others. 
Trajectories of Cancer Fears

In conclusion, group-based trajectory modeling was an effective technique to estimate distinct trajectories of longitudinal FOR. When combined with predictive models to examine associated risk factors, our findings point to psychological risk factors and coping skills to target in psychological interventions targeted to fear reduction. Future research should examine FOR over longer periods of time after diagnosis with more assessment time points and consider additional coping and social network predictors. The findings suggest that it may be a worthwhile endeavor to consider FOR a multi-dimensional construct. Although the trajectory groups and psychological correlates were more similar than different, the patterns did differ in some key ways. An understanding the domains of FOR and nature of fears and concerns may prove helpful in future research.

This article is protected by copyright. All rights reserved. 


\section{REFERENCES}

1. Ness S, Kokal J, Fee-Schroeder K, Novotny P, Satele D, Barton D. Concerns across the survivorship trajectory: results from a survey of cancer survivors. Oncol Nurs Forum. Jan 2013;40(1):35-42.

2. Savard J, Ivers $\mathrm{H}$. The evolution of fear of cancer recurrence during the cancer care trajectory and its relationship with cancer characteristics. J Psychosom Res 2013;74(4):354-360. doi: 10.1016/j.jpsychores.2012.12.013

3. Crist JV, Grunfeld EA. Factors reported to influence fear of recurrence in cancer patients: a systematic review. Psycho-oncology 2013;22(5):978-986. doi: 10.1002/pon. 3114

4. Herschbach P, Dinkel A. Fear of progression. Recent results in cancer research. Fortschritte der Krebsforschung. Progres dans les recherches sur le cancer 2014;197:11-29. doi: 10.1007/978-3-642-40187-9_2

5. Dinkel A, Kremsreiter K, Marten-Mittag B, Lahmann C. Comorbidity of fear of progression and anxiety disorders in cancer patients. Gen Hosp Psychiatry 2014;36(6):613-619. doi: 10.1016/j.genhosppsych.2014.08.006

6. Mehnert A, Berg P, Henrich G, Herschbach P. Fear of cancer progression and cancerrelated intrusive cognitions in breast cancer survivors. Psycho-oncology 2009;18(12):1273-1280. doi: 10.1002/pon.1481

This article is protected by copyright. All rights reserved. 
Trajectories of Cancer Fears

7. van den Beuken-van Everdingen MH, Peters ML, de Rijke JM, Schouten HC, van Kleef M, Patijn J. Concerns of former breast cancer patients about disease recurrence: a validation and prevalence study. Psycho-oncology 2008;17(11):1137-1145. doi: 10.1002/pon. 1340

8. Koch L, Bertram H, Eberle A, et al. Fear of recurrence in long-term breast cancer survivors-still an issue. Results on prevalence, determinants, and the association with quality of life and depression from the cancer survivorship--a multi-regional population-based study. Psycho-oncology 2014;23(5):547-554.doi: 10.1002/pon.3452

9. Kruger A, Leibbrand B, Barth J, Berger D, Lehmann C, Koch U, \& Mehnert A. [Course of psychosocial distress and health-related quality of life in patients at different age groups during cancer rehabilitation]. Zeitschrift fur Psychosomatische Medizin und Psychotherapie 2009 55(2), 141-161. doi: 10.13109/zptm.2009.55.2.141

10. Mehnert A, Koch U, Sundermann C, Dinkel A. Predictors of fear of recurrence in patients one year after cancer rehabilitation: a prospective study. Acta Oncol 2013;52(6):1102-1109.doi: 10.3109/0284186X.2013.765063

11. Melchior H, Buscher C, Thorenz A, Grochocka A, Koch U, Watzke B. Self-efficacy and fear of cancer progression during the year following diagnosis of breast cancer. Psycho-oncology 2013;22(1):39-45.doi: 10.1002/pon.2054

This article is protected by copyright. All rights reserved. 
Trajectories of Cancer Fears

12. Dunn LB, Langford DJ, Paul SM, et al. Trajectories of fear of recurrence in women with breast cancer. Supportive Care Cancer 2015;23(7):2033-2043.doi:

$10.1007 / \mathrm{s} 00520-014-2513-8$

13. McGinty HL, Small BJ, Laronga C, Jacobsen PB. Predictors and patterns of fear of cancer recurrence in breast cancer survivors. Health Psychol 2016;35(1):1-9.Health Psychology.

14. Llewellyn CD, Weinman J, McGurk M, Humphris G. Can we predict which head and neck cancer survivors develop fears of recurrence? J of Psychosom Research 2008;65(6):525-532.doi: 10.1016/j.jpsychores.2008.03.014

15. Lee-Jones C, Humphris G, Dixon R, Hatcher MB. Fear of cancer recurrence--a literature review and proposed cognitive formulation to explain exacerbation of recurrence fears. Psycho-oncology 1997;6(2):95-105.doi: Doi 10.1002/(Sici)1099-

1611(199706)6:2<95::Aid-Pon250>3.0.Co;2-B

16. Vickberg SM. The Concerns About Recurrence Scale (CARS): a systematic measure of women's fears about the possibility of breast cancer recurrence. Ann Behave Med $2003 ; 25(1): 16-24$.

17. Simard S, Savard J. Fear of Cancer Recurrence Inventory: development and initial validation of a multidimensional measure of fear of cancer recurrence. Support Care Cancer 2009;17(3):241-251.doi: 10.1007/s00520-008-0444-y

This article is protected by copyright. All rights reserved. 
18. Lebel S, Tomei C, Feldstain A, Beattie S, McCallum M. Does fear of cancer recurrence predict cancer survivors' health care use? Support Care Cancer 2013;21(3):901-906.doi: 10.1007/s00520-012-1685-3

19. Sarkar S, Scherwath A, Schirmer L, et al. Fear of recurrence and its impact on quality of life in patients with hematological cancers in the course of allogeneic hematopoietic SCT. Bone Marrow Transplant 2014;49(9):1217-1222.doi: 10.1038/bmt.2014.139

20. Simard S, Savard J, Ivers H. Fear of cancer recurrence: specific profiles and nature of intrusive thoughts. J Cancer Surviv 2010;4(4):361-371.doi: 10.1007/s11764-0100136-8

21. Liu Y, Perez M, Schootman M, Aft RL, Gillanders WE, Jeffe DB. Correlates of fear of cancer recurrence in women with ductal carcinoma in situ and early invasive breast cancer. Breast Cancer Res Treat 2011;130(1):165-173.

22. Myers SB, Manne SL, Kissane DW, et al. Social-cognitive processes associated with fear of recurrence among women newly diagnosed with gynecological cancers. Gynecol Oncol 2013;128(1):120-127.doi: 10.1016/j.ygyno.2012.10.014

23. Nagin, D.S. (2005). Group-based Modeling of Development. Cambridge, MA: Harvard University Press.

This article is protected by copyright. All rights reserved. 
Trajectories of Cancer Fears

24. D'Orazio LM, Meyerowitz BE, Stone PJ, Felix J, Muderspach LI. Psychosocial adjustment among low-income Latina cervical cancer patients. J Psychosoc Oncol 2011;29(5):515-533. doi: 10.1080/07347332.2011.599363

25. Sekse RJ, Hufthammer KO, Vika ME. Fatigue and quality of life in women treated for various types of gynaecological cancers: a cross-sectional study. J Clin Nurs 2015;24(3-4):546-55. doi: 10.1111/jocn.12647

26. Cancer Facts and Figures 2015. Retrieved March 31, 2015 http://www.cancer.org/acs/groups/content/@editorial/documents/document/acspc044552.pdf.

27. Mirabeau-Beale KL, Kornblith AB, Penson RT, et al. Comparison of the quality of life of early and advanced stage ovarian cancer survivors. Gynecol Oncol 2009;114(2):353-359.doi: DOI 10.1016/j.ygyno.2009.05.009

28. Shinn EH, Taylor CL, Kilgore K, et al. Associations with worry about dying and hopelessness in ambulatory ovarian cancer patients. Palliat Support Care 2009;7(3):299-306.doi: 10.1017/S1478951509990228

29. Lepore S. A social cognitive-processing model of emotional adjustment to cancer In A. Baum \& B. Andersen (Eds)., Psychological interventions for cancer. Washington DC: APA. 2001;99-116

30. Manne S. (unpublished data). A Pilot of a Web Based Intervention to Improve SelfCare for Oral Cancer Survivors.

This article is protected by copyright. All rights reserved. 
31. Beck A, Beamesderfer A. Assessment of depression: the depression inventory. Mod Probl Pharmacopsychiatry 1974;7(0), 151-169.

32. Horowitz M, Wilner N, Alvarez W. Impact of Event Scale: a measure of subjective stress. Psychosom Med 1979;41(3):209-218.

33. Carver CS. You want to measure coping but your protocol's too long: Consider the Brief COPE. Int J Behav Med 1997;4, 92-100.

34. King LA, Emmons RA. Conflict over Emotional Expression - Psychological and Physical Correlates. J Pers Soc Psychol 1990;58(5):864-877.

35. Telch CF, Telch MJ. Group Coping Skills Instruction and Supportive Group-Therapy for Cancer-Patients - a Comparison of Strategies. J Consult Clin Psychol 1986;54(6):802-808.

36. Manne S, Badr H, Zaider T, Nelson C, Kissane D. Cancer-related communication, relationship intimacy, and psychological distress among couples coping with localized prostate cancer. Journal of Cancer Survivorship-Research and Practice. Mar 2010;4(1):74-85.

37. Manne S, Schnoll R. Measuring supportive and unsupportive responses during cancer treatment: A factor analytic assessment of the partner responses to cancer inventory. $\mathbf{J}$ Behav Med 2001;24(4):297-321.

38. Schag C, Heinrich R. CARES: Cancer Rehabilitation Evaluation System. Santa Monica, CA: Cares Consultants. 1988. 
Trajectories of Cancer Fears

39. Jones BL, Nagin DS, Roeder K. A SAS procedure based on mixture models for estimating developmental trajectories. Sociol Method Res 2001;29(3):374-393.

40. Torbit LA, Albiani JJ, Crangle CJ, Latini DM, Hart TL. Fear of recurrence: the importance of self-efficacy and satisfaction with care in gay men with prostate cancer. Psycho-Oncology 2015;24(6):691-698.doi:10.1002/pon.3630.

41. Hayes, S.C, Strosahl, K.D., \& Wilson, K.G. (2012). Acceptance and commitment therapy: The process and practice of mindful change (2nd edition). New York, NY: The Guilford Press.

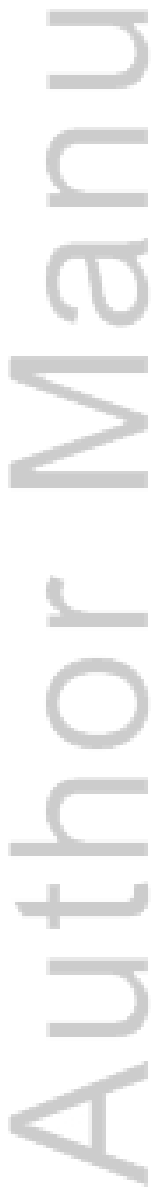

This article is protected by copyright. All rights reserved. 
Trajectories of Cancer Fears

Table 1. Demographic and medical data for study participants $(\mathrm{N}=118)$

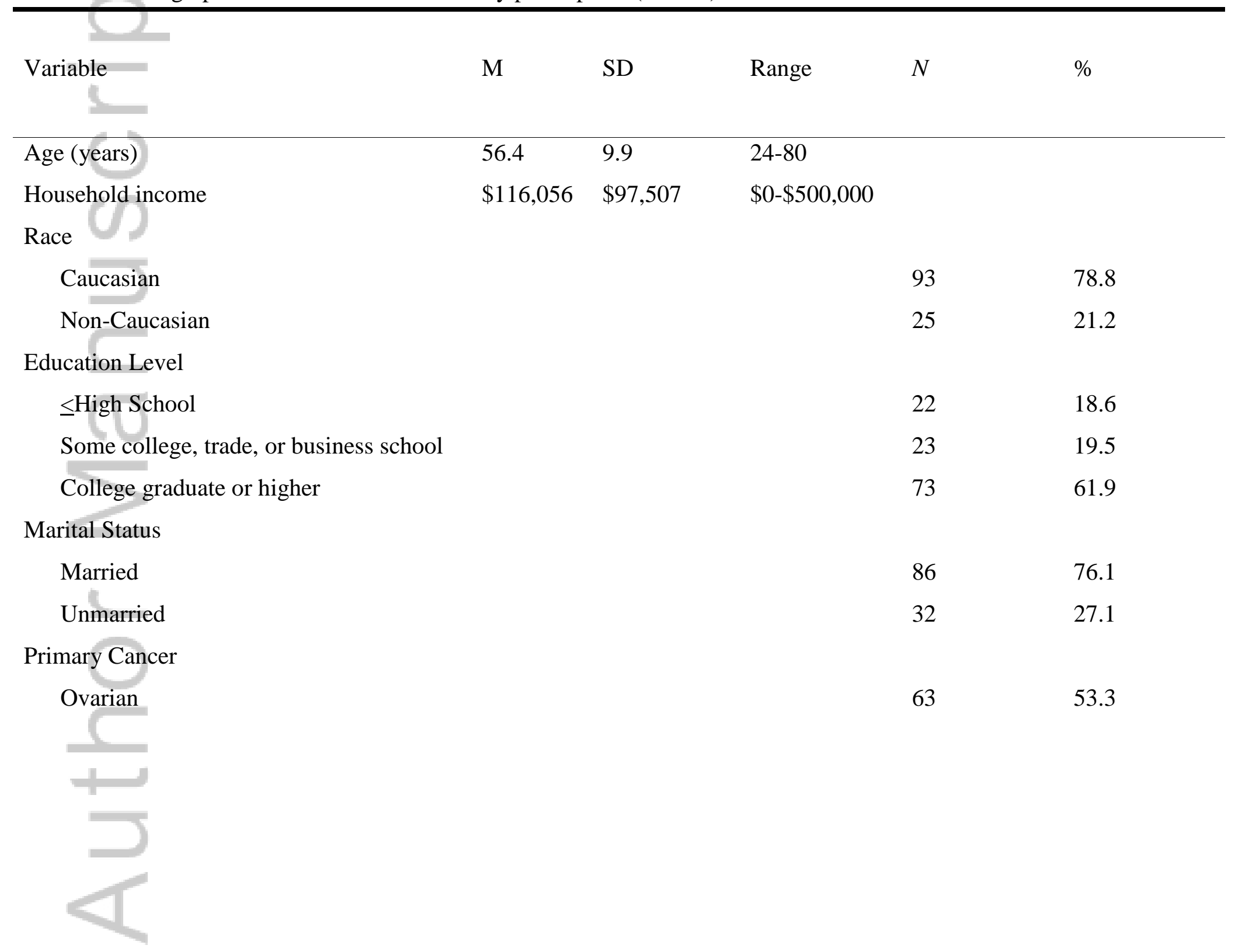

This article is protected by copyright. All rights reserved. 
Trajectories of Cancer Fears

\begin{tabular}{|c|c|c|c|c|}
\hline \multicolumn{3}{|l|}{ Endometrial } & 12 & 10.2 \\
\hline \multicolumn{3}{|l|}{ Uterine } & 7 & 5.9 \\
\hline \multicolumn{3}{|l|}{ Fallopian } & 7 & 5.9 \\
\hline \multicolumn{3}{|l|}{ Cervical } & 6 & 5.1 \\
\hline \multicolumn{3}{|l|}{ Peritoneal } & 2 & 1.7 \\
\hline \multicolumn{3}{|l|}{ More than one } & 1 & 0.84 \\
\hline \multicolumn{5}{|l|}{ Stage } \\
\hline I & & & 13 & 11.0 \\
\hline II & & & 13 & 11.0 \\
\hline III & & & 57 & 48.3 \\
\hline IV & & & 25 & 21.1 \\
\hline \multicolumn{3}{|l|}{ Metastatic site, Time 1 (yes) } & 41 & 34.7 \\
\hline Time since diagnosis (months) & 3.93 & 1.74 & & \\
\hline \multicolumn{5}{|l|}{ Treatments at Time 1} \\
\hline \multicolumn{3}{|l|}{ Surgery only } & 15 & 12.7 \\
\hline \multicolumn{3}{|l|}{ Chemotherapy only } & 3 & 2.5 \\
\hline \multicolumn{3}{|l|}{ Radiation only } & 0 & 0 \\
\hline \multicolumn{3}{|l|}{ Surgery + Chemotherapy } & 89 & 75.4 \\
\hline
\end{tabular}

This article is protected by copyright. All rights reserved. 
Trajectories of Cancer Fears

Surgery + Radiation

Surgery, Chemotherapy, + Radiation

1

0.9

Chemotherapy + radiation

-

This article is protected by copyright. All rights reserved. 
Trajectories of Cancer Fears

Table 2. Regression equations for the trajectories of global fear, role worry, health worry, and worries about womanhood over time

Variable: Global FOR

Group

1. Starts and stays low

Intercept

Time slope

Intercept

Time slope

Intercept

Time slope

$b$

se

7.808

.272

17.392

$-.966 * *$

18.587

.141
\% of sample

$.920 \quad 25.5$

.151

$1.313 \quad 25.3$

.203

.692

.150

Common Intercept SD: $\sigma=2.89 * *$

Common Residual SD: $\sigma=2.75^{* *}$

Variable: Role Worries

Group

1. Starts and stays low

2. Starts moderate and decreases

3. Starts and stays high

Common Intercept SD: $\sigma=3.11^{* *}$

Common Residual SD: $\sigma=3.38^{* *}$

Variable: Health Worries

Group

1. Starts low but increases

2. Starts high and decreases

Common Intercept SD: $\sigma=6.44 * *$

$\begin{array}{lcrc}\text { Intercept } & 5.022 & 1.206 & 33.4 \\ \text { Time slope } & .134 & .234 & \\ \text { Intercept } & 14.018 & 1.479 & 40.2 \\ \text { Time slope } & -.755^{* *} & .231 & \\ \text { Intercept } & 16.635 & 1.254 & 26.4 \\ \text { Time slope } & .294 & .234 & \end{array}$

Common Residual SD: $\sigma=6.06^{* *}$

Variable: Womanhood Worries

Group
1. Starts low and decreases
2. Starts high and increases

Intercept

Intercept
Time slope

6.452

2.628

19.1

Intercept

$.842 *$

.349

Time slope

29.018

1.013

80.9

$-.296^{*} \quad .148$

Time slope

6.708

.830

84.3

Intercept

$-.448 * *$

.124

Time slope

$17.954 \quad 2.311$

15.7

Common Intercept SD: $\sigma=5.80 * *$ 
Trajectories of Cancer Fears

Common Residual SD: $\sigma=4.51^{* *}$

Note. $* p<.05, * * p<.01$. FOR=Fear of Recurrence.

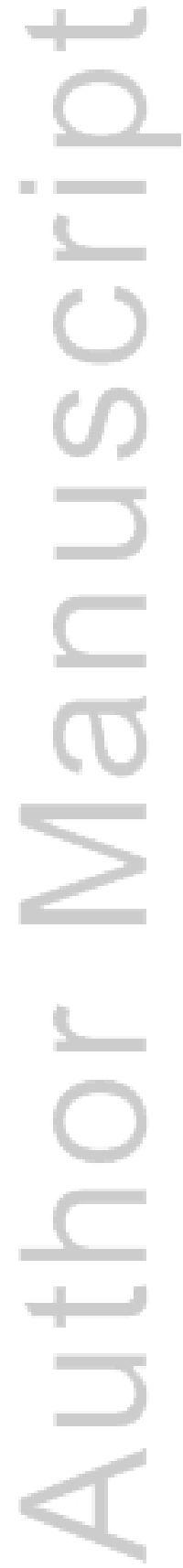

This article is protected by copyright. All rights reserved. 
Trajectories of Cancer Fears

Table 3. Means and Standard Deviations for Time 1 Vulnerability and Coping Variables as a Function of Trajectory Group for Global Fear and Role, Health, and Womanhood Worries

\begin{tabular}{|c|c|c|c|c|c|c|c|c|c|c|}
\hline$\equiv=$ & \multicolumn{3}{|c|}{$\begin{array}{c}\text { Global FOR } \\
\text { Subgroup } \\
\end{array}$} & \multicolumn{3}{|c|}{$\begin{array}{l}\text { Role Worry } \\
\text { Subgroup }\end{array}$} & \multicolumn{2}{|c|}{$\begin{array}{l}\text { Health Worry } \\
\text { Subgroup }\end{array}$} & \multicolumn{2}{|c|}{$\begin{array}{c}\text { Womanhood } \\
\text { Worry Subgroup }\end{array}$} \\
\hline & $\begin{array}{c}1 \\
M \\
(S D)\end{array}$ & $\begin{array}{c}2 \\
M \\
(S D)\end{array}$ & $\begin{array}{c}3 \\
M \\
(S D)\end{array}$ & $\begin{array}{c}1 \\
M \\
(S D)\end{array}$ & $\begin{array}{c}2 \\
M \\
(S D)\end{array}$ & $\begin{array}{c}3 \\
M \\
(S D)\end{array}$ & $\begin{array}{c}1 \\
M \\
(S D)\end{array}$ & $\begin{array}{c}2 \\
M \\
(S D)\end{array}$ & $\begin{array}{c}1 \\
M \\
(S D)\end{array}$ & $\begin{array}{c}2 \\
M \\
(S D)\end{array}$ \\
\hline Cancer-related Dist & \multicolumn{3}{|c|}{$\begin{array}{c}\mathrm{F}(2,115)=17.49 \\
p<.001\end{array}$} & \multicolumn{3}{|c|}{$\begin{array}{c}\mathrm{F}(2,115)=17.67 \\
p<.001\end{array}$} & \multicolumn{2}{|c|}{$\begin{array}{c}\mathrm{F}(1,116)=26.34 \\
p<.001\end{array}$} & $\begin{array}{c}26.43 \\
(14.90) \\
\mathrm{F}(1,116 \\
p<\end{array}$ & $\begin{array}{rl} & 45.05 \\
(13.42) \\
= & 24.49, \\
0 & 01\end{array}$ \\
\hline Depressive symptoms & \multicolumn{3}{|c|}{$\begin{array}{c}\mathrm{F}(2,115)=5.94 \\
p=.004\end{array}$} & \multicolumn{3}{|c|}{$\begin{array}{c}\mathrm{F}(2,115)=15.67 \\
p<.001\end{array}$} & $\begin{array}{c}\mathrm{F}(1,116)=14.85 \\
p<.001\end{array}$ & $\begin{array}{rl} & 14.58 \\
& (6.99) \\
= & 14.85, \\
0 & 01\end{array}$ & $\begin{array}{l}12.64 \\
(6.50) \\
\mathrm{F}(1,116 \\
p=\end{array}$ & $\begin{array}{l}18.24 \\
(9.02) \\
=9.96, \\
002\end{array}$ \\
\hline $\begin{array}{l}\text { Holding Back Sharing } \\
\text { Concerns }\end{array}$ & \multicolumn{3}{|c|}{$\begin{array}{c}\mathrm{F}(2,115)=5.43 \\
p=.006\end{array}$} & \multicolumn{3}{|c|}{$\begin{array}{c}\mathrm{F}(2,105)=7.63 \\
p=.001\end{array}$} & \multicolumn{2}{|c|}{$\begin{array}{c}\mathrm{F}(1,116)=13.37 \\
p<.001\end{array}$} & \multicolumn{2}{|c|}{$\begin{array}{c}\mathrm{F}(1,116)=5.80 \\
p=.018\end{array}$} \\
\hline Coping Self-efficacy & \multicolumn{3}{|c|}{$\begin{array}{c}\mathrm{F}(2,114)=4.74 \\
p=.010\end{array}$} & \multicolumn{3}{|c|}{$\begin{array}{c}\mathrm{F}(2,114)=5.68 \\
p=.004\end{array}$} & \multicolumn{2}{|c|}{$\begin{array}{c}\mathrm{F}(1,115)=12.31 \\
p=.001\end{array}$} & $\begin{array}{r}54.48 \\
(13.80) \\
\mathrm{F}(1,115 \\
p=\end{array}$ & $\begin{array}{l}46.67 \\
(12.08) \\
=5.32, \\
023\end{array}$ \\
\hline Positive Reappraisal & 12.20 & 9.96 & 11.26 & 11.83 & 10.43 & 11.65 & 13.45 & 10.74 & 11.17 & 11.37 \\
\hline
\end{tabular}

This article is protected by copyright. All rights reserved. 
Trajectories of Cancer Fears

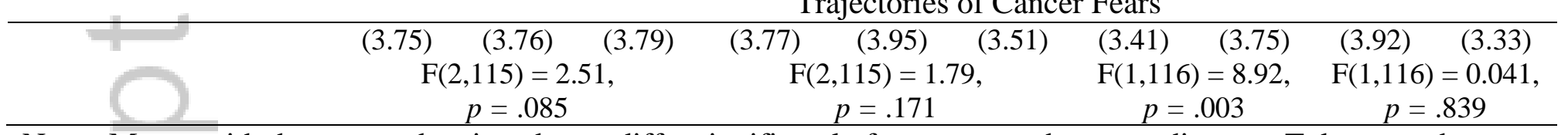

Note. Means with the same subscripts do not differ significantly from one another according to a Tukey post-hoc means test. $\mathrm{FOR}=$ Fear of Recurrence.

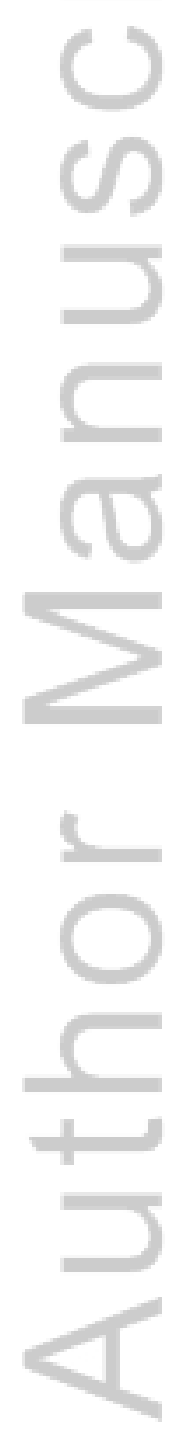

This article is protected by copyright. All rights reserved. 


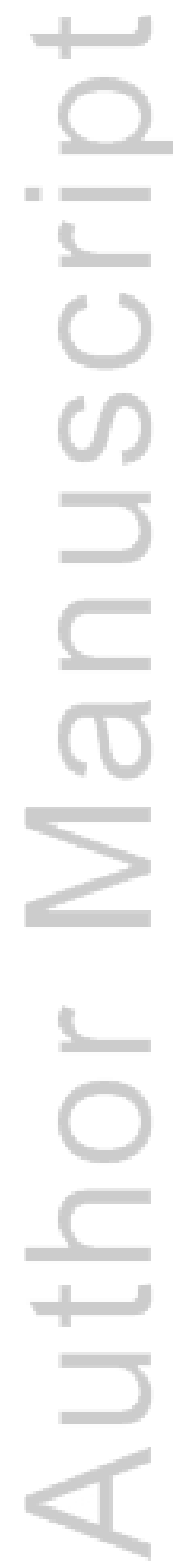

This article is protected by copyright. All rights reserved. 


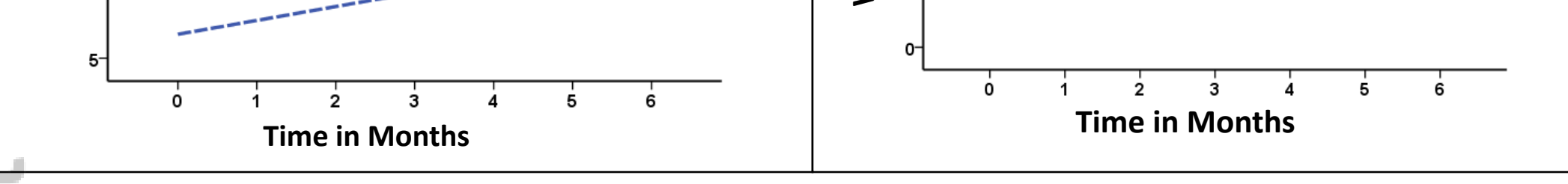

Figure 1. Trajectories of global fear, role, worry, health worry, and womanhood worry (coefficients are in Table 1).

This article is protected by copyright. All rights reserved. 


\section{University Library}

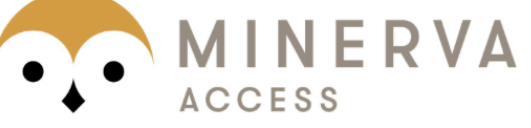

A gateway to Melbourne's research publications

Minerva Access is the Institutional Repository of The University of Melbourne

Author/s:

Manne, SL;Myers-Virtue, S;Kissane, D;Ozga, ML;Kashy, DA;Rubin, SC;Rosenblum, NG;Heckman, CJ

Title:

Group-based trajectory modeling of fear of disease recurrence among women recently diagnosed with gynecological cancers

Date:

2017-11-01

Citation:

Manne, S. L., Myers-Virtue, S., Kissane, D., Ozga, M. L., Kashy, D. A., Rubin, S. C., Rosenblum, N. G. \& Heckman, C. J. (2017). Group-based trajectory modeling of fear of disease recurrence among women recently diagnosed with gynecological cancers. PSYCHO-ONCOLOGY, 26 (11), pp.1799-1809. https://doi.org/10.1002/pon.4223.

Persistent Link:

http://hdl.handle.net/11343/291600 